\section{Testing for diabetes in hospitalised patients prescribed antipsychotic drugs}

\author{
DAVID TAYLOR, CORINA YOUNG, RAADIYYA ESOP, CAROL PATON \\ and REBECCA WALWYN
}

\begin{abstract}
Background Studies using computer databases suggest that atypical antipsychotic agents are more likely to be associated with diabetes than are conventional drugs.
\end{abstract}

Aims To discover the extent of testing for diabetes mellitus in hospital in-patients prescribed antipsychotics.

\section{Method Prescription charts were} screened to identify patients prescribed antipsychotics. Case notes were then searched for evidence of testing for diabetes.

Results In all, 606 patients were prescribed antipsychotics, of whom 250 (4I.3\%) had evidence of prior testing for diabetes. Patients prescribed atypicals were $40 \%$ more likely to have been tested than those prescribed conventional drugs $(\mathrm{RR}=1.4,95 \% \mathrm{Cl}$ I.I-1.9). Adjusted odds ratios $v$. conventional antipsychotics for testing were significantly higher for clozapine $(\mathrm{OR}=4.64,95 \% \mathrm{Cl} 2.42-8.90)$, olanzapine $(\mathrm{OR}=1.85,95 \% \mathrm{Cl}$.04-3.30) and antipsychotic polypharmacy $(\mathrm{OR}=$ 2.96, 95\% Cl I.59-5.52).

\section{Conclusions Testing for diabetes was} undertaken in less than half of the patients studied. Testing was more common in those receiving atypical antipsychotics. Apparent differences in claimed causal association of the use of some antipsychotics with diabetes may in part reflect different rates of testing.

\footnotetext{
Declaration of interest Funding from Janssen-Cilag Ltd, UK (unrestricted grant).
}

Diabetes mellitus is a growing health problem in developed countries, where its prevalence has risen substantially in the past two decades (Mokdad et al, 2000). This clearly has important consequences for health care resource use and for life expectancy (Beckman et al, 2002). Even a moderately elevated plasma glucose concentration is a continuous risk factor for myocardial infarction (Gerstein, 1999).

The prevalence of diabetes mellitus appears to be relatively high in schizophrenia (Mukherjee et al, 1996; Cassidy et al, 1999) and bipolar disorder (Dixon et al, 2000). Indeed, an association between psychotic disorders and diabetes mellitus has long been suspected, and pre-dates the widespread use of antipsychotic drugs (Braceland et al, 1946; Freeman, 1946). The introduction of conventional antipsychotic drugs appeared to increase still further the prevalence of diabetes among people with psychotic disorders (Thonnard-Neumann, 1967), and the link between the use of these drugs and diabetes became well established (Proakis et al, 1971). However, knowledge of this association seems to have dissipated to such an extent that widely used reference texts now do not list diabetes as a possible adverse effect of chlorpromazine (British Medical Association \& Royal Pharmaceutical Society of Great Britain, 2004). In recent years the use of atypical antipsychotics has been strongly linked to emergent diabetes and diabetic ketoacidosis (Mir \& Taylor, 2001). Several database studies have now established that atypical antipsychotics such as clozapine and olanzapine are more likely to be associated with a diagnosis of diabetes than are conventional drugs (Gianfrancesco et al, 2002; Koro et al, 2002; Sernyak et al, 2002).

It is important to note that the results of such studies do not directly implicate atypical antipsychotics as drugs relatively more likely to cause diabetes. Rates of diagnosis reported in these studies are dependent on screening or monitoring for (and, ultimately, identification of) diabetes. It is possible that the observed higher rate of diabetes seen with atypical drugs is at least in part a reflection of a relatively greater intensity of monitoring for diabetes when these drugs are prescribed. Such differences in monitoring practice might be expected, given the highly publicised link between atypical antipsychotic drugs and diabetes and the absence of such warnings related to conventional drugs in prescribers' reference books.

Little is known of the frequency of diabetes testing in patients with schizophrenia or related disorders or in those receiving antipsychotic treatment. We undertook a survey of the extent of diagnostic testing for diabetes in a cohort of hospitalised patients receiving a variety of antipsychotic medications.

\section{METHOD}

The study was conducted in the South London and Maudsley National Health Service (NHS) Trust and Oxleas NHS Trust during 2002 and 2003. These trusts serve a population of approximately 2 million in south-east London and semirural Kent and Surrey. Ethnic minorities form approximately $20 \%$ of the population. Ethics committee approvals were obtained for each trust.

All in-patient prescription charts were reviewed in order to identify patients prescribed antipsychotic medication. From these, patients prescribed the same antipsychotic as a regular prescription for at least 1 week were identified and included in the study.

Case notes for all patients in the study were examined (by C.Y. and R.E.) to obtain demographic data and diagnosis, and to determine whether investigations that would have enabled diagnosis of impaired fasting glucose, impaired glucose tolerance or diabetes mellitus had taken place. These investigations included measurements of fasting plasma glucose, random plasma glucose and glycosylated haemoglobin $\left(\mathrm{HbA}_{1 \mathrm{c}}\right)$ and oral glucose tolerance tests. Case note documentation of any one of these tests conducted at any time was considered to be evidence of diagnostic testing for diabetes. Recorded diagnoses of diabetes mellitus, impaired glucose tolerance or impaired fasting glucose, or prescription of oral hypoglycaemics or 
insulin, were also considered to be evidence of prior testing for diabetes. Case notes contained handwritten entries from medical and nursing staff, correspondence, chemical pathology reports and previous prescription charts. All volumes of case notes available on the ward at the time of the data retrieval exercise were examined.

Prevalence of glycaemic disorders was determined by standard diagnostic criteria. Impaired fasting glucose was diagnosed if the fasting plasma glucose level exceeded $6.1 \mathrm{mmol} / \mathrm{l}$ but was less than $7.0 \mathrm{mmol} / \mathrm{l}$. Impaired glucose tolerance was diagnosed if plasma glucose levels were greater than $7.8 \mathrm{mmol} / \mathrm{l}$ but less than $11.1 \mathrm{mmol} / \mathrm{l} 2 \mathrm{~h}$ after glucose challenge. Diabetes mellitus was diagnosed if a random plasma glucose test exceeded $11.1 \mathrm{mmol} / \mathrm{l}$, if a fasting plasma glucose test exceeded $7.0 \mathrm{mmol} / \mathrm{l}$ or if $\mathrm{HbA}_{1 \mathrm{c}}$ exceeded 9\% (National Diabetes Data Group, 1979; Lebovitz, 2001). Patients falling into more than one category on different measures were given a diagnosis of diabetes mellitus. Apparent prevalence rates were calculated by comparing the numbers diagnosed with the disorder with the number of the total sample. Statistical analysis was performed using the Statistical Package for the Social Sciences, version 10 for PC.

\section{RESULTS}

\section{Demographic factors}

In total, 606 patients satisfying inclusion criteria were identified. Of these, 250
(41.3\%) had documented evidence of testing for diabetes. Demographic details of the sample are given in Table 1.

Median age was significantly higher in those tested for diabetes (Mann-Whitney $U$ test, $Z=4.83, P<0.001)$ than in those not tested. There was no significant difference in gender distribution between those tested and those not tested $\left(\chi^{2}=2.28\right.$, d.f. $=1, P=0.13)$. Ethnicity was significantly different between the groups (Pearson's $\chi^{2}=11.06$, d.f. $=4, P=0.03$ ), with White patients overrepresented and Black patients underrepresented among those tested. Diagnosis distribution was also significantly different in the two groups (Pearson's $\chi^{2}=13.09$, d.f. $=4, \quad P=0.01$ ), with those with bipolar disorder overrepresented and those with schizophrenia underrepresented among those tested.

\section{Testing and prescribed antipsychotic}

The extent of testing of patients receiving different antipsychotic drugs is given in Table 2. There was a statistically significant association between testing for diabetes and the antipsychotic received (Pearson's $\chi^{2}=15.5$, d.f. $=5, \quad P=0.008$ ). Overall, 389 patients received an atypical antipsychotic drug as their sole antipsychotic treatment. Of these, 168 $(43.2 \%)$ were tested for diabetes. The risk ratio for testing was 1.4 (95\% CI 1.1-1.9; $P=0.005)$, compared with testing in patients receiving conventional antipsychotics.

\section{Prevalence of glycaemic disorder}

Of the 250 patients tested, 2 were classified as having impaired fasting glucose and 37 as having diabetes mellitus; the remaining 211 patients had no evidence of a disorder of glucose homoeostasis (test results normal). None was diagnosed with impaired glucose tolerance. Thus, 39 patients had evidence of glucose homoeostasis disorder, a prevalence of $15.6 \%$ in those tested. The apparent prevalence of glucose homoeostasis disorder in the total study population (based on number of diagnosed cases) was $6.4 \%$ (39 of 606 ). The numbers of diagnosed cases for each drug treatment are shown in Table 3. Prevalence of glucose homoeostasis disorder was not significantly associated with treatment for those tested $\quad\left(n=250 ; \quad \chi^{2}=7.216, \quad\right.$ d.f. $=5$, $P=0.205)$ or for the total sample $(n=606$; $\chi^{2}=6.470$, d.f. $=5, P=0.263$ ). 
Table 3 Prevalence of glycaemic disorders

\begin{tabular}{|c|c|c|c|c|c|c|}
\hline \multirow[t]{2}{*}{ Treatment } & \multirow{2}{*}{$\begin{array}{c}\text { Patients } \\
\text { tested } \\
n\end{array}$} & \multicolumn{3}{|c|}{ Cases of glycaemic disorders } & \multirow{2}{*}{$\begin{array}{c}\text { Prevalence in } \\
\text { those tested } \\
\%\end{array}$} & \multirow{2}{*}{$\begin{array}{c}\text { Apparent prevalence } \\
\text { in sample } \\
\%\end{array}$} \\
\hline & & IFG $n$ & $\mathrm{DM} n$ & Total IGT/DM n & & \\
\hline Conventional antipsychotics & 38 & 0 & 10 & 10 & 26.3 & 8.1 \\
\hline Clozapine & 49 & 0 & 8 & 8 & 16.3 & 9.1 \\
\hline Olanzapine & 60 & 0 & 6 & 6 & 10.0 & 4.1 \\
\hline Risperidone & 46 & 0 & 4 & 4 & 8.7 & 3.3 \\
\hline Other atypical antipsychotics & 13 & 0 & 2 & 2 & 15.4 & 5.7 \\
\hline Antipsychotic polypharmacy & 44 & 2 & 7 & 9 & 20.5 & 9.7 \\
\hline Total & 250 & 2 & 37 & 39 & 15.6 & 6.4 \\
\hline
\end{tabular}

DM, diabetes mellitus; IFG, impaired fasting glucose; IGT, impaired glucose tolerance.

\section{Duration of antipsychotic treatment}

The duration of treatment with the antipsychotic prescribed at the time of the study was determined for 579 patients $(96 \%)$. The mean duration of treatment was 12.2 months (range 0.23-228, s.d.=24.9; figures rounded up to nearest week). In those tested for diabetes, mean treatment duration was 16.2 months (range $0.23-228$, s.d. $=30.2$ ) and median 4.0 months. In patients not tested, mean duration of treatment was 9.4 months (range $0.23-174$, s.d. $=20.1$ ) and median 3.0 months. The duration of treatment with different drugs is shown in Table 4 .

\section{Odds of testing for diabetes}

Because likelihood of testing was associated with factors other than drug prescribed, we calculated adjusted odds ratios for diabetes testing for the different drug treatments (compared with testing for conventional antipsychotics), taking into account age, gender, ethnicity, diagnosis and duration of treatment (Table 5).

Table 4 Duration of treatment with drug prescribed at the time of the study

\begin{tabular}{lcrrr}
\hline \multirow{2}{*}{ Treatment } & Number of patients & \multicolumn{3}{c}{ Duration, months } \\
\cline { 3 - 5 } & & Mean & Median & Range \\
\hline Conventional antipsychotics & 114 & 17.8 & 4.0 & $0.23-228$ \\
Clozapine & 80 & 22.0 & 8.5 & $0.23-135$ \\
Olanzapine & 141 & 8.9 & 3.0 & $0.23-108$ \\
Risperidone & 119 & 5.1 & 2.0 & $0.23-99$ \\
Other atypical antipsychotics & 34 & 4.3 & 2.0 & $0.23-23$ \\
Antipsychotic polypharmacy & 91 & 13.9 & 4.0 & $0.23-140$ \\
\hline
\end{tabular}

Table 5 Odds ratios for diabetes testing

\begin{tabular}{|c|c|c|c|c|}
\hline \multirow[t]{2}{*}{ Treatment } & \multicolumn{2}{|c|}{ Unadjusted odds ratio } & \multicolumn{2}{|c|}{ Adjusted odds ratio' } \\
\hline & Estimate & $95 \% \mathrm{Cl}$ & Estimate & $95 \% \mathrm{Cl}$ \\
\hline Conventional antipsychotics & 1.00 & & 1.00 & \\
\hline Clozapine & 2.84 & $\mathrm{I} .6 \mathrm{I}-5.02$ & 4.64 & $2.42-8.90$ \\
\hline Olanzapine & 1.60 & $0.96-2.65$ & 1.85 & $1.04-3.30$ \\
\hline Risperidone & 1.39 & $0.82-2.36$ & 1.68 & $0.92-3.08$ \\
\hline Other atypical antipsychotics & 1.34 & $0.6 \mathrm{I}-2.93$ & 1.95 & $0.81-4.71$ \\
\hline Antipsychotic polypharmacy & 2.03 & $1.16-3.55$ & 2.96 & I.59-5.52 \\
\hline
\end{tabular}

I. Adjusted for age, gender, ethnicity, diagnosis and treatment duration.

\section{DISCUSSION}

The two major findings of this study are that testing for diabetes had been undertaken in less than half of the patients receiving antipsychotics and that testing was more frequently done in those receiving atypical antipsychotics than in those receiving conventional drugs. These findings have important consequences for the interpretation of diabetes prevalence studies using health care databases.

\section{Consequences}

The major consequence of testing such a small proportion of patients is that the overall prevalence of diabetes mellitus is likely to be underestimated. Nearly threefifths of patients had never been tested for diabetes, and it is likely that this conceals a number of patients with undiagnosed disease. This supposition is supported by a study showing high rates of hyperglycaemia and diabetes mellitus in patients receiving clozapine who had no previous diagnosis of diabetes (Sernyak et al, 2003). This is likely to mean that many cases of diabetes will go unnoticed and untreated, with serious or even fatal consequences for patients. In this study cohort, it is possible that those with symptoms or risk factors for diabetes were tested for the disorder but those considered not to be at risk were not tested. Fewer cases of diabetes would then have been missed. It is also important to note that 63 patients had received the antipsychotic prescribed at the time of the study for less than 1 month (although many had received other antipsychotics before) a period during which drug-induced diabetes might not be expected. Although these factors are worthwhile considerations 
and possible mitigating factors, it remains highly probable that many cases of disordered glucose homoeostasis were missed because of incomplete testing in this cohort. Indeed, another indicator of the paucity of monitoring for diabetes is the absence of diagnoses of impaired glucose tolerance a reflection of the limited number of oral glucose tolerance tests conducted in this cohort.

\section{Variation in testing}

The variation in the extent of testing for diabetes with the type of antipsychotic prescribed suggests that its diagnosis might be more likely in some patients at least partly because of more intensive testing. In this study, testing was significantly more likely to have occurred in patients taking clozapine, more than one antipsychotic drug and atypical drugs in general. Testing was numerically more frequent in all patients receiving individual atypical drugs compared with those receiving conventional drugs. This occurred despite the mean treatment duration being longer with conventional drugs than with all other agents except clozapine. Indeed, adjusted odds of testing, taking into account all other influencing factors, were significantly higher for clozapine, olanzapine and polypharmacy. Although these findings suggest clinicians are now aware of the risk of diabetes with atypical antipsychotics, differential use of testing will inevitably mean that the overall prevalence of diabetes is likely to be underestimated to a greater extent in those prescribed conventional rather than atypical antipsychotics. This is amply demonstrated by the differences in prevalence figures between those tested and in the total sample (see Table 3): apparent prevalence figures conceal major differences in diabetes prevalence.

\section{Comparison with prior studies}

If such differences in testing or screening are repeated in other secondary care units, as suggested by Boilson \& Hamilton (2003), other clinical environments (e.g. primary care) and other countries, then interpretation of the results of database studies must inevitably be modified. The message from these studies (Gianfrancesco et al, 2002, 2003; Koro et al, 2002; Sernyak et al, 2002) is that diabetes mellitus is more frequently associated with atypical antipsychotic therapy than with conventional drugs; the implication is that atypical drugs

\section{CLINICAL IMPLICATIONS}

- Database studies are likely to underestimate the true prevalence of diabetes mellitus in patients taking antipsychotics.

- More intensive screening for diabetes in patients on atypical antipsychotics may in part explain the apparent higher prevalence of diabetes seen with these drugs.

- All patients receiving antipsychotics should undergo regular testing for diabetes.

\section{LIMITATIONS}

- The study sample may not be representative of other patient groups in the UK and elsewhere.

- Higher rates of testing in patients on atypical drugs may reflect a higher frequency of symptoms.

- Case note searching may not have revealed details of all prior testing for diabetes.

DAVID TAYLOR, PhD, MRPharmS, CORINA YOUNG, BPharm, MRPharmS, Pharmacy, Maudsley Hospital, London; RAADIYYA ESOP, BPharm, MRPharmS, CAROL PATON, MCMHP, MRPharmS, Pharmacy, Bexley Hospital, Bexley; REBECCA WALWYN, BSc, MSc, Clinical Trials Unit, Institute of Psychiatry, London, UK

Correspondence: Dr David Taylor, Pharmacy Department, Maudsley Hospital, London SE5 8AZ, UK. Tel: +44(0)20 7740 5040; fax: +44(0)20 7919 3448; e-mail: David.Taylor@slam.nhs.uk

(First received 3 December 2003, final revision 16 April 2004, accepted 22 April 2004)

are relatively more likely to cause diabetes. This may only be assumed, however, if the intensity of testing is similar for all drugs. None of the studies cited above measured screening rates and few mentioned this factor as a possible confounding variable. Few accounted for other possible influences such as age, ethnicity, diagnosis and duration of treatment. So, it is entirely possible that database studies of antipsychotics and diabetes mellitus inadvertently overestimate the differences in causal association between individual antipsychotics, and may erroneously link relatively higher rates of diabetes with atypical drugs.

Also of note in our study is the relatively higher prevalence of documented testing among White patients and patients with bipolar disorder. Only 36\% (82 of $228)$ of participants of Black African descent had been tested and only 37\% (155 of 419) of those with schizophrenia or schizoaffective disorder. These are important and damning observations, given the high risk of diabetes in these patient groups.

None the less, it is possible that our method provided an incomplete view of testing for diabetes in these patients. We searched all case notes available on hospital wards at the time of our visit. Many patients have several volumes of case notes and it is possible that some volumes were missing from the ward. We cannot discount the possibility that some patients had undergone tests for diabetes and that the results of these tests appeared in missing case notes and were not alluded to in notes available to us. Tests might also have been conducted and recorded in the community. It is unlikely, however, that a diagnosis of diabetes mellitus would be concealed in this way, since once recorded either in hospital or general practice notes the diagnosis would be likely to be mentioned in correspondence or case histories contained in current notes available to us. Moreover, data collected on duration of treatment indirectly indicate the substantial length of time covered by our case note search.

In conclusion, testing for diabetes in patients prescribed antipsychotics was incomplete. The paucity of testing for a condition that has considerable morbidity and that is strongly associated with 
increased mortality is cause for concern, especially since effective treatments are available. Clearly, treatments cannot be prescribed if the disorder is not diagnosed. In addition, the variation in the extent of testing with drug prescribed casts doubt over database study findings which suggest increased risks with atypical drugs.

\section{ACKNOWLEDGEMENT}

We thank Professor A. H. Barnett of the Department of Medicine, University of Birmingham, for advice on this study.

\section{REFERENCES}

Beckman, J. A., Creager, M. A. \& Libby, P. (2002)

Diabetes and atherosclerosis: epidemiology,

pathophysiology, and management. JAMA, 287 2570-2581.

Boilson, M. \& Hamilton, R. J. (2003) A survey of monitoring of weight and blood glucose in in-patients. Psychiatric Bulletin, 27, 424-426.

Braceland, F. J., Meduna, L. J. \& Vaichulis, J. A. (1946) Delayed action of insulin in schizophrenia. American journal of Psychiatry, 102, 108-III.

British Medical Association \& Royal

Pharmaceutical Society of Great Britain (2004)
British National Formulary (March issue). London \& Wallingford: BMJ Books \& Pharmaceutical Press.

Cassidy, F., Ahearn, E. \& Carroll, B. J. (1999) Elevated frequency of diabetes mellitus in hospitalized manicdepressive patients. American Journal of Psychiatry, 156 1417-1420.

Dixon, L., Weiden, P., Delahanty, J., et al (2000)

Prevalence and correlates of diabetes in national schizophrenia samples. Schizophrenia, 26, 903-912.

Freeman, H. (1946) Resistance to insulin in mentally disturbed soldiers. Archives of Neurology and Psychiatry, 56, 74-77.

Gerstein, H. C. (1999) Relationship of glucose and insulin levels to the risk of myocardial infarction: casecontrol study. Journal of the American College of Cardiology, 33, 612-619.

Gianfrancesco, F. D., Grogg, A. L., Mahmoud, R. A., et al (2002) Differential effects of risperidone, olanzapine, clozapine, and conventional antipsychotics on type 2 diabetes: findings from a large health plan database. Journal of Clinical Psychiatry, 93, 920-929.

Gianfrancesco, F. D., White, R., Wang, R., et al (2003) Antipsychotic-induced type 2 diabetes: evidence from a large health plan database. Journal of Clinical Psychopharmacology, 23, 328-335.

Koro, C. E., Fedder, D. O., L'Italien, G. J., et al (2002) Assessment of independent effect of olanzapine and risperidone on risk of diabetes among patients with schizophrenia: population based nested case-control study. BMJ, 532, 243-245.
Lebovitz, H. E. (200I) Diagnosis, classification and pathogenesis of diabetes mellitus. Journal of Clinical Psychiatry, 62, 5-9.

Mir, S. \& Taylor, D. (200I) Atypical antipsychotics and hyperglycaemia. International Clinical Psychopharmacology, 16, 63-74

Mokdad, A. H., Ford, E. S., Bowman, B. A., et al (2000) Diabetes trends in the US: 1990-1998. Diabetes Care, 3, 1278-1283.

Mukherjee, S., Decina, P., Bocoloa, V., et al (1996) Diabetes mellitus in schizophrenic patients. Comprehensive Psychiatry, 34, 68-73.

National Diabetes Data Group (1979) Classification and diagnosis of diabetes mellitus and other categories of glucose intolerance. Diabetes, 28, 1039-1057.

Proakis, A. G., Mennear, J. H., Miya, T. S., et al (197I) Phenothiazine-induced hyperglycaemia: relation to CNS and adrenal effects. Proceedings of the Society of Experimental Biology and Medicine, 137, 1385-1388.

Sernyak, M. J., Leslie, D. L., Alarcon, R. D., et al (2002) Association of diabetes mellitus with use of atypical neuroleptics in the treatment of schizophrenia. American Journal of Psychiatry, 157, 561-566.

Sernyak, M. J., Gulanski, B., Leslie, D. L., et al (2003) Undiagnosed hyperglycaemia in clozapine-treated patients with schizophrenia. Journal of Clinical Psychiatry, 64, 605-608.

Thonnard-Neumann, E. (1967) Phenothiazines and diabetes in hospitalised women. American Journal of Psychiatry, 124, 978-981. 